\title{
Monitoramento da broca-pequena-do-fruto para tomada de decisão de controle em tomateiro estaqueado
}

\author{
Sérgio Roberto Benvenga ${ }^{1}$; Sergio Antonio De Bortoli²; Santin Gravena ${ }^{1}$; José C Barbosa ${ }^{3}$ \\ ${ }^{1}$ Gravena-Pesquisa, Consultoria e Treinamento Agrícola Ltda., C. Postal 546, 14870-990 Jaboticabal-SP; ${ }^{2}$ UNESP-FCAV, Lab. Biologia \\ e Criação de Insetos; Rod. Prof. Paulo Donato Castellane, s/n, 14884-900 Jaboticabal-SP; ${ }^{3}$ UNESP-FCAV, Dep ${ }^{\text {to }}$ Ciências Exatas; srben- \\ venga@gravena.com.br; bortoli@fcav.unesp.br; jcbarbosa@fcav.unesp.br
}

\section{RESUMO}

Avaliou-se a relação entre o índice de plantas com ovos e de adultos da broca-pequena-do-fruto capturados em armadilhas com feromônio sexual, bem como a influência da infestação na produção da cultura do tomate. Estas avaliações objetivaram aperfeiçoar a tomada de decisão de controle pela densidade de adultos capturados nas armadilhas. Em Monte Mor, estado de São Paulo, áreas comerciais de cultivo de tomate foram divididas em parcelas experimentais para a instalação de armadilhas. Em intervalos médios de 3,7 dias foi avaliada a densidade de adultos capturados e a infestação de plantas com ovos nos frutos. O estudo foi conduzido entre as safras de 2003 e 2007 para os cultivos em ciclo de verão e inverno. As avaliações foram realizadas até o término da colheita e foi definida a produção comercializada e a descartada. O controle químico foi realizado quando a infestação atingia o nível de $5 \%$ de plantas com ovos nos frutos. A pressão populacional foi mais expressiva nos cultivos em ciclo de verão. $\mathrm{O}$ aumento no número de machos capturados nas armadilhas com feromônio sexual correspondeu a um incremento na infestação de ovos nos frutos e houve influência positiva da infestação de plantas com a produção descartada. A instalação das armadilhas deve anteceder o florescimento e a tomada de decisão de controle ocorrer no prazo médio de oito dias após a captura média de 0,24 e 0,23 adultos na armadilha por dia para os cultivos de tomate em ciclo de verão e inverno, respectivamente.

Palavras-chave: Neoleucinodes elegantalis, Solanum lycopersicon, armadilha, feromônio, correlação, nível de ação, manejo integrado de pragas.

\begin{abstract}
Monitoring the tomato fruit borer population for the control decision

The relation between the number of plants with eggs and the number of tomato fruit borer adults trapped in sexual pheromone traps and, also, the influence of infestation on the tomato crop production were evaluated. Commercial tomato fields in Monte Mor, São Paulo State, were divided into plots for the installation of traps. With an interval of 3.7 days, the density of trapped adults and the fruit infestation with eggs were evaluated. The study was carried out between 2003 and 2007 seasons for crops in Summer and Winter cycles. The evaluations were carried out until the end of the harvest and commercial and discarded production were evaluated. The chemical pest control was carried out when the infestation reached $5 \%$ of plants with eggs on fruits. The population was more significant in Summer cycle crops. The increase of the number of males trapped in sexual pheromone traps corresponded to an increase in the infestation of eggs on fruits and infestation influenced positively the discarded production. The installation of the traps must be done before the flowering stage and the control should be performed about eight days after the registration of an average of 0.24 and 0.23 adult per trap per day for the summer and winter cycles, respectively.
\end{abstract}

Keywords: Neoleucinodes elegantalis, Solanum lycopersicon, trap, pheromone, correlation, action level, integrated pest management.

\section{(Recebido para publicação em 23 de setembro de 2009; aceito em 1 de setembro de 2010) (Received on September 23, 2009; accepted on September 1, 2010)}

A b roca-pequena-do-fruto $\mathrm{NeO}$ leucinodes elegantalis (Guenée) (Lepidoptera: Crambidae), é classificada como praga-chave na cultura do tomate, Lycopersicon esculentum, devido ao dano direto ocasionado no fruto. Prejuízos de 50 a $90 \%$ da produção foram relatados (Nunes \& Leal, 2001; Gallo et al., 2002; Gravena \& Benvenga, 2003; Miranda et al., 2005).

As fêmeas apresentam o comportamento de oviposição seletiva, depositando os ovos na superfície dos frutos em início de desenvolvimento (Blackmer et al., 2001; Rodrigues Filho et al., 2003; Gravena \& Benvenga, 2003). As lagartas recém-eclodidas apresentam um curto período de trânsito na superfície do fruto até localizarem o ponto de entrada, preferencialmente na porção medianainferior do fruto (Eiras \& Blackmer, 2003). No interior dos frutos as lagartas permanecem protegidas das ações de controle e alimentam-se do endocarpo, caracterizando o dano da praga, remanescendo os orifícios de saída ao término do período larval.

A fisiologia do tomateiro, de apresentar floradas sucessivas e frutos em fase inicial de desenvolvimento, aliada ao comportamento de oviposição e desenvolvimento larval garantem a combinação hospedeiro:praga ideal para desencadear o início da reprodução. Desta forma, o controle químico destaca-se como a principal tática de controle. Em casos extremos, são realizadas até três aplicações por semana, a partir do início do florescimento, para o controle de $N$. elegantalis (Badji et al., 2003; Miranda et al., 2005).

Para o manejo da broca-pequenado-fruto, Gravena \& Benvenga (2003) recomendam a divisão da cultura em talhões de um hectare, com inspeção de 60 plantas em 12 pontos casualizados, duas vezes por semana. O método de amostragem preconizado é o de avaliar visualmente as pencas no terço superior contendo frutos em fase inicial de desenvolvimento. Este método apresenta 
como vantagem a facilidade de observação das posturas na superfície dos frutos, permitindo a tomada de decisão de controle na fase de maior suscetibilidade da praga (Eiras \& Blackmer, 2003; Rodrigues Filho et al., 2003).

Gravena \& Benvenga (2003) utilizaram o método de avaliação de ovos da broca-pequena-do-fruto em pesquisa e nos cultivos comerciais, sendo o rendimento definido em função do nível de detalhamento exigido na avaliação das partes reprodutivas das plantas. Além disso, esse método exige o treinamento de um profissional na inspeção de pragas quanto ao reconhecimento das diferentes fases do ciclo de desenvolvimento de $N$. elegantalis e de outros agentes fitófagos para que as informações geradas sejam tecnicamente seguras para a tomada de decisão de controle.

Neste sentido, novas tecnologias devem ser pesquisadas, como a utilização de armadilhas contendo o feromônio sintético para a amostragem do nível populacional de adultos, considerado como o primeiro indício da presença da praga na cultura, visando estabelecer métodos rápidos, práticos e confiáveis, valorizando, assim, o manejo integrado de pragas (Bento, 2000).

Para adequar a armadilha de feromônio como um método seguro de amostragem da broca-pequena-do-fruto é necessária a comparação com a infestação nas plantas. Entretanto, ainda não foi definida a relação entre o número de adultos de $N$. elegantalis capturados nas armadilhas e o índice de infestação da praga na planta, ou mesmo a influência da infestação sobre a produtividade da cultura, para definir a medida de controle. Assim, estudou-se a relação entre a infestação de $N$. elegantalis na planta e adultos na armadilha com feromônio, a densidade de adultos na armadilha para tomada de decisão de controle e a influência da infestação sobre a produtividade da cultura do tomate estaqueado.

\section{MATERIAL E MÉTODOS}

$\mathrm{O}$ estudo foi realizado em Monte Mor-SP (22 ${ }^{\circ} 56^{\prime} 48^{\prime}$ 'S, $47^{\circ} 18^{\prime} 57^{\prime \prime} \mathrm{W}, 560$ $\mathrm{m}$ de altitude), em áreas comerciais de tomate, com o híbrido Alambra, sob sistema estaqueado de condução. Os ensaios foram realizados nos cultivos de verão e de inverno, respectivamente, iniciando-se na safra do ano de 2003 e prosseguindo-se a experimentação até a safra do ano de 2007.

As áreas comerciais de cultivo com aproximadamente 15 ha foram divididas em parcelas experimentais de um ha, com densidade média de 17.440 plantas, para a posterior distribuição das armadilhas de feromônio.

A amostragem, quanto à infestação por broca-pequena-do-fruto, foi realizada regularmente em 60 plantas por parcela (cinco plantas em 12 pontos casualizados), ao longo dos carreadores divisórios (bordadura). Utilizou-se o método de visualizar as pencas com frutos em fase inicial de desenvolvimento, a partir do ápice das plantas, considerando-se infestadas aquelas que apresentavam pelo menos um fruto com ovos.

Em cada parcela experimental foram instaladas duas armadilhas do tipo Delta para o monitoramente de adultos com um septo de látex impregnado com o feromônio sexual $\mathrm{BIO} \mathrm{NEO}^{\circledR}$ (Bio Controle, Métodos de controle de pragas Ltda., São Paulo-SP), composto de E 11-hexadecen-1-ol e Z3, Z6, Z9-tricosatrieno $(0,023 \%)$ e inertes (polipropileno) $(99,977 \%)$. As armadilhas foram devidamente identificadas e posteriormente distribuídas nos vértices opostos de cada parcela experimental, fixadas a uma haste de bambu de 1,5 $\mathrm{m}$ de altura, para a manutenção sempre acima do porte das plantas, de acordo com o estágio fenológico. Os septos de feromônio foram substituídos a cada 45 dias e os fundos adesivos descartáveis das armadilhas, em intervalo semanal, de acordo com a recomendação da empresa registrante. Os septos foram fixados com auxílio de arame na parte superior da armadilha.

Em média, a amostragem nos cultivos de verão foi iniciada em 3 de março e encerrada em 20 de junho, com a lavoura apresentando 16 e 125 dias de transplantio, respectivamente, totalizando 29 avaliações. Nos cultivos de inverno foram realizadas 26 amostragens entre 28 de agosto e 1 de dezembro, com a lavoura apresentando
18 e 113 dias de transplantio, respectivamente. A amostragem das plantas e o monitoramento das armadilhas foram realizados nas mesmas datas. Nos cultivos de tomate em ciclo de verão o intervalo médio entre as avaliações foi de 3,75 dias e de 3,65 dias, para os cultivos em ciclo de inverno. Assim, para uniformizar os valores referentes à densidade de adultos nas armadilhas visando definir o número mínimo de insetos acumulados para a tomada de decisão de controle, estabeleceu-se o número médio de adultos capturados diariamente na armadilha.

Os resultados de infestação de $N$. elegantalis nos frutos serviram de base para as tomadas de decisão de controle químico nas parcelas experimentais com o índice superior ou igual a 5\% de plantas com ovos nos frutos, considerado como o nível de ação referencial em cultivos sob orientações de manejo integrado de pragas (Gravena \& Benvenga, 2003). Utilizaram-se os inseticidas registrados para o manejo da praga na cultura, preconizando-se a rotação de produtos com distintos mecanismos de ação para o correto manejo de resistência (Omoto, 2000).

Para avaliar a influência da infestação da broca-pequena-do-fruto nas plantas e a densidade de adultos nas armadilhas sobre a produtividade da cultura foi realizada a colheita individualizada por parcela nas áreas experimentais em cultivo de verão. Ao término do cultivo nas áreas experimentais foi contabilizada a produção comercializada e a produção descartada, respectivamente, por parcela experimental, sendo os valores expressos em caixas de $24 \mathrm{~kg} / 1000$ plantas cultivadas.

$\mathrm{O}$ índice de plantas infestadas com ovos de $N$. elegantalis foi correlacionado com o número de insetos capturados nas armadilhas por dia visando à definição da densidade de adultos para a tomada de decisão de controle nos cultivos de verão e inverno, respectivamente. Realizou-se análise de regressão para as variáveis adultos e avaliação da infestação nas plantas, utilizando-se o procedimento PROC CORR (SAS Institute Inc., Cary, NC, USA, versão 9). Realizou-se também a correlação entre a infestação na planta e o número de 
adultos nas armadilhas, com a produção comercializada e a produção descartada, respectivamente, com o objetivo de avaliar o impacto da ocorrência da praga sobre a produtividade da cultura.

\section{RESULTADOS E DISCUSSÃO}

Infestação e densidade populacional - A amostragem das plantas utilizando-se do método de visualização das pencas com frutos em fase inicial de desenvolvimento indicou que nos cultivos de verão o índice de plantas com ovos de $N$. elegantalis nos frutos é superior ao verificado nos cultivos de tomate em ciclo de inverno (Tabela 1). A média ponderada do índice de plantas com ovos nos frutos foi de 10,94\% e $2,70 \%$ para os cultivos de verão e inverno, respectivamente. As avaliações que contribuíram para destacar os cultivos de verão como os de maior pressão populacional da praga foram aquelas realizadas em 2004 e 2007, cujos índices de infestação foram 13,80\% e 14,22\%, respectivamente, diferindo dos verificados nos demais períodos de avaliação. Análise semelhante foi realizada para os cultivos de inverno, indicando que as avaliações realizadas em 2006, com índice de infestação de plantas com ovos de $0,70 \%$, contribuíram de forma significativa, em relação aos outros períodos de avaliação, para a confirmação dos cultivos de inverno como aqueles de menor pressão populacional da praga.

A densidade de adultos capturados em armadilha por dia nos cultivos de tomate em ciclo de verão foi de 0,44 inseto e superou o verificado nas armadilhas instaladas nos cultivos de inverno, cujo valor diário foi de 0,16 inseto por armadilha. A maior captura diária de insetos nas armadilhas instaladas na cultura do tomate em ciclo de verão ocorreu no ano de 2005, diferindo do verificado nos demais períodos de avaliação. Em 2005, o número médio de insetos capturados por dia foi de 0,70; no ano de 2006 o valor diário não superou 0,25 inseto por armadilha, correspondendo ao período de menor captura de adultos da broca-pequena-do-fruto. Ainda, no ano de 2005, também foi verificado o maior número de insetos capturados diariamente nas armadilhas no cultivo

Tabela 1. Incidência de plantas infestadas com ovos nos frutos e densidade de adultos de Neoleucinodes elegantalis capturados nas armadilhas com o feromônio sexual BIO NEO ${ }^{\circledR}$, em cultivos comerciais de tomate estaqueado (incidence of plants infested with eggs on fruits and Neoleucinodes elegantalis adults trapped in sexual pheromone traps in commercial tomato fields). Monte Mor-SP, Gravena, 2003-2007.

\begin{tabular}{|c|c|c|c|c|}
\hline \multicolumn{5}{|c|}{ Índice médio de plantas com ovos nos frutos ${ }^{1 / 3}$} \\
\hline \multirow{2}{*}{ Ano } & \multicolumn{2}{|c|}{ Verão } & \multicolumn{2}{|c|}{ Inverno } \\
\hline & (n) & Média \pm EP & (n) & Média \pm EP \\
\hline 2003 & 442 & $9,62 \pm 0,56 \mathrm{Ab}$ & 364 & $3,73 \pm 0,20 \mathrm{Ba}$ \\
\hline 2004 & 392 & $13,80 \pm 0,78 \mathrm{Aa}$ & 336 & $2,53 \pm 0,19 \mathrm{Ba}$ \\
\hline 2005 & 338 & $8,91 \pm 0,41 \mathrm{Ab}$ & 372 & $2,76 \pm 0,20 \mathrm{Ba}$ \\
\hline 2006 & 464 & $8,33 \pm 0,45 \mathrm{Ab}$ & 325 & $0,70 \pm 0,08 \mathrm{Bb}$ \\
\hline 2007 & 420 & $14,22 \pm 0,76 \mathrm{Aa}$ & 325 & $3,65 \pm 0,28 \mathrm{Ba}$ \\
\hline Média & 411 & $10,94 \pm 0,60 \mathrm{~A}$ & 344 & $2,70 \pm 0,19 \mathrm{~B}$ \\
\hline \multicolumn{5}{|c|}{ Número médio de adultos capturados na armadilha por dia $^{2 / 3}$} \\
\hline \multirow{2}{*}{ Ano } & \multicolumn{2}{|c|}{ Verão } & \multicolumn{2}{|c|}{ Inverno } \\
\hline & (n) & Média \pm EP & (n) & Média \pm EP \\
\hline 2003 & 442 & $0,36 \pm 0,09 \mathrm{Ac}$ & 364 & $0,15 \pm 0,02 \mathrm{Ba}$ \\
\hline 2004 & 392 & $0,42 \pm 0,05 \mathrm{Ac}$ & 336 & $0,19 \pm 0,04 \mathrm{Ba}$ \\
\hline 2005 & 338 & $0,70 \pm 0,12 \mathrm{Aa}$ & 372 & $0,23 \pm 0,03 \mathrm{Ba}$ \\
\hline 2006 & 464 & $0,25 \pm 0,04 \mathrm{Ad}$ & 325 & $0,05 \pm 0,01 \mathrm{Bb}$ \\
\hline 2007 & 420 & $0,53 \pm 0,09 \mathrm{Ab}$ & 325 & $0,18 \pm 0,04 \mathrm{Ba}$ \\
\hline Média & 411 & $0,44 \pm 0,07 \mathrm{~A}$ & 344 & $0,16 \pm 0,02 \mathrm{~B}$ \\
\hline
\end{tabular}

${ }^{1}$ Dados originais. Para a análise estatística foram transformados em $\operatorname{arc} \operatorname{sen}\left[(\mathrm{p} / 100)^{1 / 2}\right] ;{ }^{2} \mathrm{Dados}$ originais. Para a análise estatística foram transformados em $\left[(\mathrm{x}+0,5)^{1 / 2}\right] ;{ }^{3} \mathrm{Nas}$ linhas, médias seguidas de mesma letra maiúscula e nas colunas, seguidas de mesma letra minúscula, não diferem entre si por Tukey $(\mathrm{p}>0,05)$ ( ${ }^{1}$ original data transformed through $\operatorname{arc} \operatorname{sen}\left[(\mathrm{p} / 100)^{1 / 2}\right]$ only for the statistical analysis; ${ }^{2}$ original data transformed through $\left[(x+0,5)^{1 / 2}\right]$ only for the statistical analysis; ${ }^{3}$ On lines, values followed by the same capital letters and, in columns, the ones which are followed by the same lowercase letters are not different by the Tukey's test $(\mathrm{p}>0,05))$.

de inverno, com valor de 0,23 adulto, superando apenas o observado no ano de 2006, com 0,05 adulto na armadilha por dia.

Relação entre insetos nas armadilhas e infestação nas plantas - $\mathrm{O}$ número de adultos na armadilha por dia proporcionou correlação significativa e positiva da incidência de plantas infestadas com ovos em todas as safras de avaliação dos cultivos de tomate em ciclo de verão (Tabela 2). A mesma correlação foi verificada entre os parâmetros de infestação da broca-pequena-do-fruto em todas as safras de avaliação dos cultivos de tomate em ciclo de inverno. Estes resultados referem-se à análise de regressão entre a densidade de adultos nas armadilhas e infestação nas plantas para a mesma data de avaliação, não sendo estabelecido um lapso de tempo entre a detecção de machos nas armadilhas e a deposição de ovos nas plantas.

O número médio de adultos na armadilha por dia a cada data de avaliação dos cultivos de tomate em ciclo de verão e inverno, respectivamente, foram correlacionados com a incidência de plantas infestadas com ovos e estão expressos na Tabela 4, ilustrando a regressão entre os parâmetros de infestação de $N$. elegantalis.

Os parâmetros de infestação obtidos com a instalação de armadilhas com o feromônio sexual resultaram em relação linear e positiva com a ocorrência de plantas infestadas com ovos nos cultivos de tomate em ambas as estações. Os coeficientes de determinação referentes ao número de adultos na armadilha por dia em relação à porcentagem de plantas com ovos nos cultivos em ciclo de verão foram mais elevados quando 
Tabela 2. Coeficientes de correlação linear $(r)$ e parâmetros das regressões ajustadas (y = a $+\mathrm{bx})$ com coeficientes de determinação $\left(\mathrm{R}^{2}\right)$ entre a densidade de adultos de Neoleucinodes elegantalis capturados nas armadilhas com o feromônio sexual BIO NEO ${ }^{\circledR}$ e a incidência de plantas infestadas com ovos nos frutos, em cultivos comerciais de tomate estaqueado (linear correlation coefficients $(r)$ and parameters of the adjusted regressions $(y=a+b x)$ with their coefficients of determination $\left(\mathrm{R}^{2}\right)$ between the density of Neoleucinodes elegantalis adults in sexual pheromone traps and incidence of tomato plants infested with eggs). Monte MorSP, Gravena, 2003-2007.

\begin{tabular}{|c|c|c|c|c|c|c|c|c|}
\hline \multicolumn{9}{|c|}{$\mathrm{N}^{0}$ adultos na armadilha por dia $\mathrm{x}$ plantas com ovos $(\%)^{1}$} \\
\hline \multirow{2}{*}{ Ano } & \multicolumn{4}{|c|}{ Verão } & \multicolumn{4}{|c|}{ Inverno } \\
\hline & $\mathbf{r}$ & $\mathbf{a}$ & $\mathbf{b}$ & $\mathbf{R}^{2}$ & $\mathbf{r}$ & $\mathbf{a}$ & $\mathbf{b}$ & $\mathbf{R}^{2}$ \\
\hline 2003 & $0,72 * *$ & 0,147 & 0,021 & 0,43 & $0,47 * *$ & 0,084 & 0,018 & 0,34 \\
\hline 2004 & $0,41 * *$ & 0,271 & 0,010 & 0,21 & $0,51 * *$ & 0,083 & 0,042 & 0,46 \\
\hline 2005 & $0,56^{* *}$ & 0,296 & 0,045 & 0,38 & $0,35^{* *}$ & 0,148 & 0,030 & 0,33 \\
\hline 2006 & $0,34^{* *}$ & 0,177 & 0,008 & 0,26 & $0,39 * *$ & 0,022 & 0,033 & 0,36 \\
\hline 2007 & $0,58 * *$ & 0,150 & 0,026 & 0,49 & $0,34 * *$ & 0,069 & 0,031 & 0,31 \\
\hline
\end{tabular}

1; significativo ao nível de $5 \%$;**significativo ao nível de $1 \%(1 ; *$ significant at $5 \%$; **significant at $1 \%$ ).

Tabela 3. Coeficientes de correlação linear $(r)$ e parâmetros das regressões ajustadas $(y=a$ + bx) com coeficientes de determinação $\left(\mathrm{R}^{2}\right)$ entre os parâmetros de infestação de Neoleucinodes elegantalis e a produtividade da cultura do tomate estaqueado em cultivos comerciais de verão (linear correlation coefficients $(r)$ and parameters of the adjusted regressions $(\mathrm{y}=$ $\mathrm{a}+\mathrm{bx}$ ) with their coefficients of determination $\left(\mathrm{R}^{2}\right)$ between the parameters of Neoleucinodes elegantalis infestation and summer cycle tomato crops productivity). Monte Mor-SP,

Gravena, 2003-2007.

\% plantas com ovos x produção (n ${ }^{0}$ caixas $24 \mathrm{~kg} / 1.000$ plantas) ${ }^{1}$

\begin{tabular}{|c|c|c|c|c|c|c|c|c|}
\hline \multirow{2}{*}{ Ano } & \multicolumn{4}{|c|}{ Comercializada } & \multicolumn{4}{|c|}{ Descartada } \\
\hline & $\mathbf{r}$ & $\mathbf{a}$ & b & $\mathbf{R}^{2}$ & $\mathbf{r}$ & $\mathbf{a}$ & b & $\mathbf{R}^{2}$ \\
\hline 2003 & $-0,18 * *$ & 39,65 & $-0,073$ & 0,53 & $0,15^{\mathrm{ns}}$ & $-0,572$ & 3,897 & 0,35 \\
\hline 2004 & $-0,30 * *$ & 76,30 & $-0,229$ & 0,78 & $0,27 * *$ & 3,976 & 1,664 & 0,66 \\
\hline 2005 & $-0,39 * *$ & 54,76 & $-0,140$ & 0,71 & $0,35 * *$ & 4,778 & 1,855 & 0,56 \\
\hline 2006 & $-0,52 * *$ & 52,64 & $-0,229$ & 0,90 & 0,40 & 4,963 & 1,770 & 0,54 \\
\hline 2007 & $-0,26 * *$ & 73,85 & $-0,202$ & 0,86 & $0,25 * *$ & 7,352 & 1,160 & 0,81 \\
\hline
\end{tabular}

$\mathrm{N}^{0}$ adultos na armadilha por dia $x$ produção ( $\mathrm{n}^{0}$ caixas $24 \mathrm{~kg} / 1.000$ plantas) ${ }^{1}$

\begin{tabular}{llcccccccc}
\hline \multirow{2}{*}{ Ano } & \multicolumn{4}{c}{ Comercializada } & & \multicolumn{4}{c}{ Descartada } \\
\cline { 2 - 3 } \cline { 7 - 9 } & $\mathbf{r}$ & $\mathbf{a}$ & $\mathbf{b}$ & $\mathbf{R}^{2}$ & & $\mathbf{r}$ & $\mathbf{a}$ & $\mathbf{b}$ & $\mathbf{R}^{\mathbf{2}}$ \\
\hline $\mathbf{2 0 0 3}$ & $-0,08^{\text {ns }}$ & 1,071 & $-0,001$ & 0,27 & & $0,05^{\text {ns }}$ & 0,176 & 0,069 & 0,10 \\
$\mathbf{2 0 0 4}$ & $-0,08^{\text {ns }}$ & 0,864 & $-0,001$ & 0,07 & & $0,15^{\text {ns }}$ & 0,273 & 0,024 & 0,28 \\
$\mathbf{2 0 0 5}$ & $-0,17^{\text {ns }}$ & 2,846 & $-0,006$ & 0,29 & & $0,12^{\text {ns }}$ & 0,541 & 0,070 & 0,15 \\
$\mathbf{2 0 0 6}$ & $-0,14^{\text {ns }}$ & 0,680 & $-0,002$ & 0,32 & & $0,09^{\text {ns }}$ & 0,219 & 0,014 & 0,13 \\
$\mathbf{2 0 0 7}$ & $-0,19^{* *}$ & 2,332 & $-0,006$ & 0,54 & & $0,15^{\text {ns }}$ & 0,363 & 0,028 & 0,33 \\
\hline
\end{tabular}

1;* significativo ao nível de $5 \%$; ** significativo ao nível de $1 \%\left({ }^{1 ; *}\right.$ significant at $5 \%$; **significant at $1 \%$ ).

comparados com os mesmos parâmetros nos cultivos de inverno.

Relação entre os parâmetros de infestação e a produtividade - $\mathrm{O}$ índice de plantas infestadas com ovos resultou em correlação significativa e negativa com a produção comercializada em todas as safras de tomate em ciclo de verão (Tabela 3). Correlação significativa e positiva foi verificada entre $o$ descartada e os parâmetros de infestação da broca-pequena-do-fruto nas armadilhas com feromônio sexual.

O índice médio de plantas com ovos em cada parcela experimental dos cultivos em ciclo de verão foi correlacionado com a produção descartada e está expresso na Tabela 5, ilustrando a regressão entre a infestação de plantas com ovos e a produtividade nos cultivos, sob o sistema de manejo integrado de pragas.

As avaliações periódicas das plantas quanto à presença de ovos nos frutos em fase inicial de desenvolvimento resultaram em relação linear e positiva com a produção descartada nos cultivos de verão. $\mathrm{O}$ mesmo parâmetro de infestação nas plantas resultou em relação linear e negativa com a produção comercializada. Entretanto, somente o coeficiente de determinação referente ao índice de plantas com ovos e a produção descartada nos cultivos de verão foi significativo.

A maior incidência de plantas infestadas com ovos de $N$. elegantalis nos frutos e de adultos nas armadilhas com o feromônio sexual foi verificada nas áreas experimentais da cultura do tomate em ciclo de verão. O mesmo resultado foi encontrado por Jordão \& Nakano (2000), Nunes \& Leal (2001) e Miranda et al. (2005), confirmando os cultivos de verão como os de maior pressão populacional deste lepidóptero-praga.

Segundo Marcano (1991a, b), a viabilidade do ciclo de vida é dependente da combinação de fatores climáticos, sendo favorecida com umidade relativa superior a $65 \%$ e temperatura máxima de $25^{\circ} \mathrm{C}$. Assim, compreende-se melhor os resultados quanto à maior pressão populacional nos cultivos em ciclo de verão, concordando com os dados obtidos por Fernández et al. (1988) quanto à maior incidência da praga nos períodos de maiores índices pluviométricos, ressaltando a importância da umidade relativa na viabilidade do ciclo de vida do inseto.

A correlação significativa e positiva do número de adultos na armadilha por dia com a incidência de plantas infestadas com ovos, utilizando-se o método proposto por Gravena \& Benvenga (2003), em todas as safras de avaliação 
Tabela 4. Regressão linear entre a densidade de adultos de Neoleucinodes elegantalis capturados nas armadilhas com o feromônio sexual BIO NEO ${ }^{\circledR}$ e a incidência de plantas infestadas com ovos nos frutos, em cultivos comerciais de tomate estaqueado, sob o sistema de manejo integrado de pragas (linear regression between the density of Neoleucinodes elegantalis adults trapped in sexual pheromone traps and incidence of tomato plants infested with eggs). Monte Mor-SP, Gravena, 2003-2007.

\begin{tabular}{|c|c|c|c|}
\hline \multicolumn{2}{|c|}{$\begin{array}{c}\text { Ciclo de verão } \\
\mathrm{y}=\mathbf{0 , 0 3 6 x}+\mathbf{0 , 0 5 7}\left(\mathbf{R}^{2}=\mathbf{0 , 9 4 * *}\right)\end{array}$} & \multicolumn{2}{|c|}{$\begin{array}{c}\text { Ciclo de inverno } \\
\mathrm{y}=\mathbf{0 , 0 2 9 x}+\mathbf{0 , 0 8 5}\left(\mathbf{R}^{2}=0,82 * *\right)\end{array}$} \\
\hline $\begin{array}{l}\text { Plantas com } \\
\text { ovos }(\%)\end{array}$ & $\begin{array}{c}\text { Adultos na } \\
\text { armadilha por dia }\left(\mathrm{N}^{0}\right)\end{array}$ & $\begin{array}{l}\text { Plantas com } \\
\text { ovos }\end{array}$ & $\begin{array}{l}\text { Adultos na armadilha } \\
\text { por dia }\left(\mathbf{N}^{\circ}\right)\end{array}$ \\
\hline 0,0 & 0,06 & 0,0 & 0,09 \\
\hline 2,5 & 0,15 & 1,0 & 0,11 \\
\hline 5,0 & 0,24 & 2,0 & 0,14 \\
\hline 7,5 & 0,33 & 3,0 & 0,17 \\
\hline 10,0 & 0,42 & 4,0 & 0,20 \\
\hline 12,5 & 0,51 & 5,0 & 0,23 \\
\hline 15,0 & 0,60 & 6,0 & 0,26 \\
\hline 17,5 & 0,69 & 7,0 & 0,29 \\
\hline 20,0 & 0,78 & 8,0 & 0,32 \\
\hline 25,0 & 0,96 & 9,0 & 0,35 \\
\hline 30,0 & 1,14 & 10,0 & 0,38 \\
\hline
\end{tabular}

Tabela 5. Regressão linear entre a infestação de Neoleucinodes elegantalis e a produtividade da cultura do tomate estaqueado em cultivos comerciais de verão, sob o sistema de manejo integrado de pragas (linear regression between Neoleucinodes elegantalis infestation and summer cycle tomato crops productivity). Monte Mor-SP, Gravena, 2003-2007.

\begin{tabular}{ccc}
\hline \multicolumn{3}{c}{$\begin{array}{c}\text { Produção descartada } \\
\mathbf{y}=\mathbf{1 , 4 5 4} \mathbf{+}+\mathbf{5 , 5 7 6}\left(\mathbf{R}^{\mathbf{2}}=\mathbf{0 , 6 8 * *}\right)\end{array}$} \\
\hline $\begin{array}{c}\text { Produção descartada } \\
\left(\mathbf{n}^{\mathbf{0}} \text { caixas 24 kg/1.000 plantas) }\right.\end{array}$ & $\begin{array}{c}\text { Plantas com } \\
\text { ovos (\%) }\end{array}$ & $\begin{array}{c}\text { Adultos na armadilha } \\
\text { por dia }\left(\mathbf{n}^{\mathbf{0}}\right)\end{array}$ \\
\hline 0,0 & 5,58 & 0,26 \\
1,0 & 7,03 & 0,31 \\
2,0 & 8,48 & 0,36 \\
3,0 & 9,94 & 0,41 \\
4,0 & 11,39 & 0,47 \\
5,0 & 12,85 & 0,52 \\
6,0 & 14,30 & 0,57 \\
7,0 & 15,75 & 0,62 \\
8,0 & 17,21 & 0,68 \\
9,0 & 18,66 & 0,73 \\
10,0 & 20,12 & 0,78 \\
\hline
\end{tabular}

dos cultivos de tomate em ciclo de verão e inverno, indica que as armadilhas podem ser adotadas para a amostragem do nível populacional de $N$. elegantalis, visando estabelecer métodos rápidos, práticos e confiáveis para a tomada de decisão de controle (Bento, 2000).

Os resultados de correlação foram para este fato é que a cópula ocorre mais comumente para aqueles com um dia de emergência (Marcano, 1991a; Jaffe et al., 2007). Além disso, o período de pré-oviposição desta espécie é de aproximadamente 2,7 dias (Marcano, 1991a). Desta forma, ao ser realizada a captura de machos nas armadilhas, um curto período de tempo é estimado para a visualização de ovos nos frutos. $\mathrm{O}$ fato de os adultos serem os primeiros indicativos da presença da praga na cultura ressalta as vantagens do monitoramento com o uso de armadilhas com feromônio sexual, pois o produtor teria um prazo médio de 8,8 dias para realizar o controle químico, equivalente ao intervalo entre o acasalamento e a eclosão das lagartas na superfície dos frutos, pois o menor período de incubação é de 5,1 dias a $25^{\circ} \mathrm{C}$ (Marcano, 1991a), favorecendo, assim, a eficiência de controle dos inseticidas. Além disso, definindose as fases infestantes da praga podem ser priorizados inseticidas específicos e com características de seletividade aos agentes de controle biológico (Gravena \& Benvenga, 2003).

Nos cultivos de tomate em ambientes abertos podem ser verificadas gerações sobrepostas de $N$. elegantalis devido à migração e à emergência diária de adultos. Desta forma, é necessário estabelecer uma densidade referencial de adultos capturados diariamente nas armadilhas com correspondente perda estimada, para que seja utilizada como nível de ação para a tomada de decisão de controle.

O índice de plantas infestadas com ovos resultou em correlação significativa e positiva com a produção descartada nas safras de 2004 a 2007 dos cultivos de tomate em ciclo de verão. Desta forma, com o incremento na infestação de plantas com ovos ocorre maior descarte de frutos, pois a influência da infestação com ovos nos frutos sobre a produção foi expressa por uma equação linear e positiva.

A correlação entre a produção comercializada e o número de adultos por armadilha e de adultos na armadilha por dia, respectivamente, não foi significativa em todas as safras de avaliação dos cultivos de tomate em ciclo de verão. O mesmo resultado foi verificado para a análise de regressão entre a produção 
descartada e os parâmetros de infestação da broca-pequena-do-fruto nas armadilhas com feromônio sexual. A correlação não significativa entre estes parâmetros está diretamente relacionada aos aspectos bioecológicos da praga, pois os frutos tornam-se impróprios para o comércio e processamento industrial por apresentarem a polpa destruída (Gallo et al., 2002) e, muitas vezes, também infectada por patógenos a partir do orifício de saída da lagarta (Gravena \& Benvenga, 2003). Portanto, o potencial de dano é caracterizado por apenas uma lagarta no interior do fruto. Desta forma, como as armadilhas coletam seletivamente os machos, era esperado que não houvesse influência da população de adultos nas armadilhas e a redução da produtividade. Além disso, como mencionado anteriormente, é necessário um intervalo de tempo após a captura de adultos nas armadilhas para ocorrer a oviposição e a eclosão das lagartas, havendo influência do controle químico realizado nas áreas experimentais aos níveis de ação preconizados por Gravena \& Benvenga (2003).

A utilização das armadilhas com feromônio sexual para o monitoramento de adultos de $N$. elegantalis apresenta, sobre a avaliação direta da presença de ovos nos frutos, a vantagem da maior rapidez na obtenção dos resultados, bastando realizar as avaliações em intervalos pré-definidos, fazendo-se a substituição dos fundos e dos septos de acordo com a recomendação do fabricante. Além disso, é de fácil adoção por parte dos produtores, pois o simples reconhecimento dos aspectos morfológicos dos adultos capturados já os tornam habilitados a fazer o monitoramento da população da praga. Adotando-se o nível de ação sugerido por Gravena \& Benvenga (2003), que é de 5\% de plantas com ovos nos frutos em fase inicial de desenvolvimento, como a infestação referencial para a tomada de decisão de controle, o mesmo nível de ação utilizando-se das armadilhas seria de 0,24 adultos na armadilha por dia para os cultivos em ciclo de verão e 0,23 adultos na armadilha por dia, para os cultivos em ciclo de inverno. Realizando-se o controle químico quando for atingida esta densidade referencial de adultos nas armadilhas a produção descartada por $N$. elegantalis nos cultivos em ciclo de verão é nula. Como a produção descartada apresenta relação direta com a infestação de plantas com ovos e, consequentemente, com a densidade de adultos capturados, foi estabelecida uma escala de produção descartada com equivalente infestação por ovos e adultos, respectivamente. Desta forma, havendo oscilação do valor de venda da produção comercializada, o produtor pode assumir uma perda estimada e adotar outro nível de ação referencial, com influência direta na intensidade de aplicação de inseticidas, garantindo a lucratividade do agronegócio.

Portanto, a contribuição científica deste trabalho está relacionada ao detalhamento da infestação de $N$. elegantalis em plantas, a correspondente infestação em armadilhas e a influência sobre a produtividade da cultura do tomate. Assim, um novo campo de pesquisa sobre a viabilidade econômica da cultura do tomate em ciclo de verão sob influência da broca-pequena-do-fruto pode ser implementado, adotando-se níveis de ação fixos para a tomada de decisão de controle e a contabilidade do número de aplicações realizadas, com a correspondente produção descartada, permitindo-se estabelecer níveis de ação compatíveis com a lucratividade, gerando um menor impacto ambiental e ganhos nutricionais para o consumidor.

\section{AGRADECIMENTOS}

À Gravena Ltda. pelo suporte técnico e financiamento da pesquisa; ao produtor Lázaro Lauro de Andrade pela concessão da área comercial e aos inspetores de pragas Vanderlei Souza Lesse e Eldimar Francisco de Souza, pelo auxílio nas coletas de frutos em campo.

\section{REFERÊNCIAS}

BADJI CA; EIRAS AE; CABRERA A; JAFFE K. 2003. Avaliação do feromônio sexual de Neoleucinodes elegantalis Guenée (Lepidoptera: Crambidae). Neotropical Entomology 32: 221-229.

BENTO JMS. 2000. Controle de insetos por comportamento: feromônios. In: GUEDES JC; COSTA ID; CASTIGLIONI E. (eds). Bases e técnicas do manejo de insetos. Santa Maria:
UFSM/CCR/DFS, p. 85-97.

BLACKMER JL; EIRAS AE; SOUZA CLM. 2001. Oviposition preference of Neoleucinodes elegantalis (Guenée) (Lepidoptera: Crambidae) and rates of parasitism by Trichogramma pretiosum Riley (Hymenoptera: Trichogrammatidae) on Lycopersicon esculentum in São José de Ubá-RJ, Brazil. Neotropical Entomology 30: 89-95.

EIRAS AE; BLACKMER JL. 2003. Eclosion time and larval behavior of the tomato fruit borer, Neoleucinodes elegantalis (Guenée) (Lepidoptera: Crambidae). Scientia Agricola 60: 195-197.

FERNÁNDEZ S; SALAS J; ALVAREZ C; PARRA A. 1988. Fluctuacion poblacional de los principales insectos-plaga del tomate em la depresion de Quibor, Estado Lara, Venezuela. Agronomia Tropical 37: 31-42.

GALLO D; NAKANO O; SILVEIRA NETO S; CARVALHO RPL; BAPTISTA GC; BERTI FILHO E; PARRA JRP; ZUCCHI RA; ALVES SB; VENDRAMIM JD; MARCHINI LC; LOPES JRS; OMOTO C. 2002. Entomologia agrícola. Piracicaba: FEALQ, p. 757-769.

GRAVENA S; BENVENGA SR. 2003. Manual prático para manejo de pragas do tomate. Jaboticabal: Gravena Ltda. 144 p.

JAFFE K; MIRÁS B; CABRERA A. 2007. Mate selection in the moth Neoleucinodes elegantalis: evidence for a supernormal chemical stimulus in sexual attraction. Animal Behaviour 73: 727-734.

JORDÃO AL; NAKANO O. 2000. Controle de lagartas dos frutos do tomateiro pelo ensacamento das pencas. Anais da Sociedade Entomológica do Brasil 29: 773-782.

MARCANO R. 1991a. Estudio de la biologia y algunos aspectos del comportamiento del perforador del fruto del tomate Neoleucinodes elegantalis (Lepidoptera: Pyralidae) en tomate. Agronomia Tropical 41: 257-263.

MARCANO R. 1991b. Ciclo biologico del perfurador del fruto del tomate Neoleucinodes elegantalis (Guenée) (Lepidoptera: Pyralidae), usando berenjena (Solanum melongena) como alimento. Boletin de Entomologia Venezolana 6: 135-141.

MIRANDAMMM; PICANÇO MC; ZANUNCIO JC; BACCI L; SILVA EM. 2005. Impact of integrated pest management on the population of leafminers, fruit borers, and natural enemies in tomato. Ciência Rural 35: 204-208.

NUNES MUC; LEAL MLS. 2001. Efeito da aplicação de biofertilizante e outros produtos químicos e biológicos, no controle da brocapequena do fruto e na produção do tomateiro tutorado em duas épocas de cultivo e dois sistemas de irrigação. Horticultura Brasileira 19: 53-59.

OMOTO C. 2000. Modo de ação de inseticidas e resistência de insetos a inseticidas. In: GUEDES JC; COSTA ID; CASTIGLIONI E. (eds). Bases e técnicas do manejo de insetos. Santa Maria: UFSM/CCR/DFS. p. 31-50.

RODRIGUES FILHO IL; MARCHIOR LC; SILVA LV. 2003. Análise da oviposição de Neoleucinodes elegantalis (Guén., 1854) (Lep.: Crambidae) para subsidiar estratégia de manejo. Agronomia 37: 23-26. 